\title{
Measurements of the Higgs boson decaying into two photons at CMS
}

\author{
Edward Scott* on behalf of the CMS Collaboration \\ Imperial College London \\ E-mail: ed.scott@eern.ch
}

\begin{abstract}
The latest results of the Higgs boson decaying to two photons at the CMS experiment are presented. Measurements are based on the $35.9 \mathrm{fb}^{-1} 2016$ dataset at a centre-of-mass energy of $13 \mathrm{TeV}$. An overall signal strength of $1.18_{-0.14}^{+0.17}$ relative to the Standard Model prediction is observed. Signal strengths of different production processes, couplings to fermions and bosons, and effective couplings to photons and gluons are also reported.
\end{abstract}

The 39th International Conference on High Energy Physics (ICHEP2018)

4-11 July, 2018

Seoul, Korea

${ }^{*}$ Speaker. 


\section{Introduction}

Despite its small branch ratio of around $0.2 \%$, the diphoton channel was one of the most important channels for the discovery of the Higgs boson in 2012, and continues to be a sensitive channel wellsuited to the precise characterisation of Higgs properties. The CMS experiment [1] has analysed $35.9 \mathrm{fb}^{-1}$ of 2016 data at a centre-of-mass energy of $13 \mathrm{TeV}$ [2]. This facilitates a wide range of measurements, including inclusive and per-process signal strengths, coupling modifiers to fermions and bosons, and effective coupling modifiers to photons and gluons.

\section{Analysis Strategy}

In order to maximise the sensitivity of the analysis, and enable discrimination between production processes, events are categorised into several categories. The four Untagged categories comprise mostly gluon fusion ( $\mathrm{ggH}$ ) events and are defined using a diphoton BDT, whose inputs are photon kinematics and identification variables. The Vector Boson Fusion (VBF) process is targeted by identifying events with two additional jets, and using a dijet BDT to reduce the contamination from ggH events. The dijet BDT has jet kinematic variables as inputs, and is used together with the diphoton BDT to form the combined BDT used to define the three VBF categories. There are two top quark pair associated $(\mathrm{ttH})$ categories: the BDT-based hadronic tag and the cut-based leptonic tag. Finally, there are five associated production $(\mathrm{VH})$ categories targeting hadronic and leptonic $\mathrm{W}$ and $\mathrm{Z}$ boson decays. Full details of the event categorisation and their validation can be found in [2]. The resulting mass distribution, with the fitted signal and background components, is shown in Figure 1.
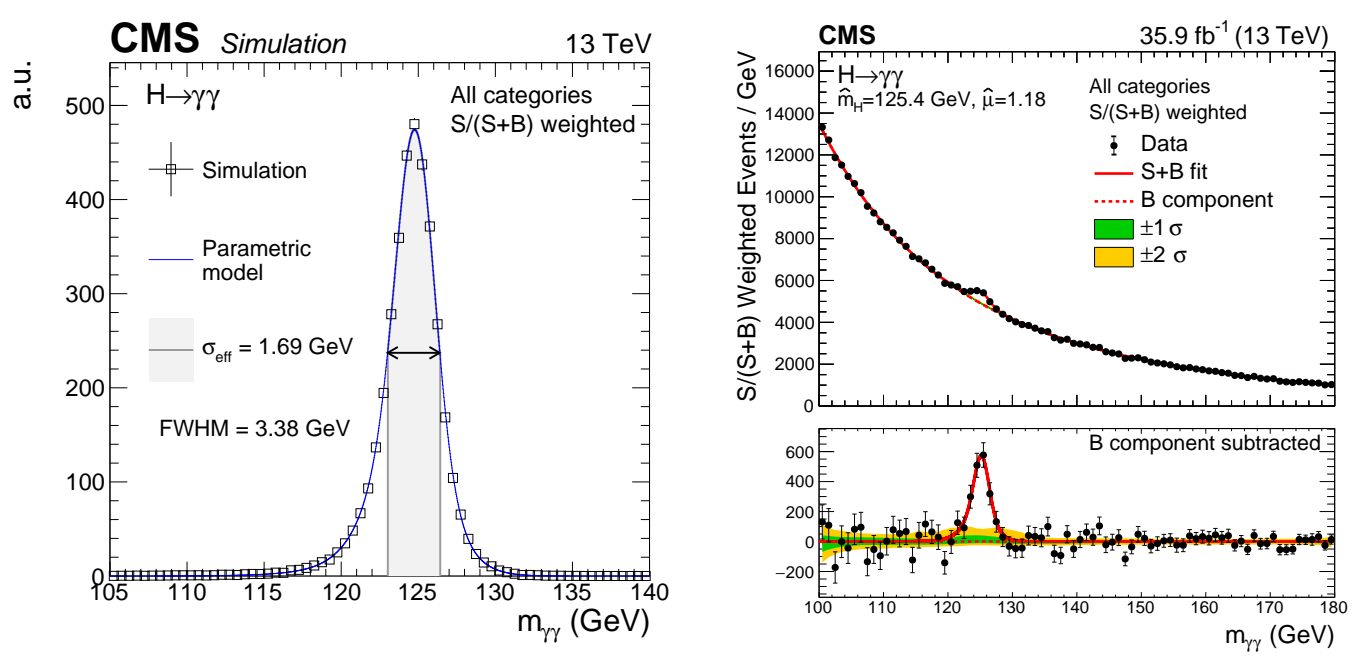

Figure 1: Left: Parametrised signal shape for all categories combined together and weighted by the $S /(S+B)$ ratio for a simulated $\mathrm{H} \rightarrow \gamma \gamma$ signal sample with $m_{H}=125 \mathrm{GeV}$. Right: Data and signal-plus-background model fits where the categories are summed weighted by their sensitivity. The one (green) and two (yellow) standard deviation bands include the uncertainties in the background component of the fit. 

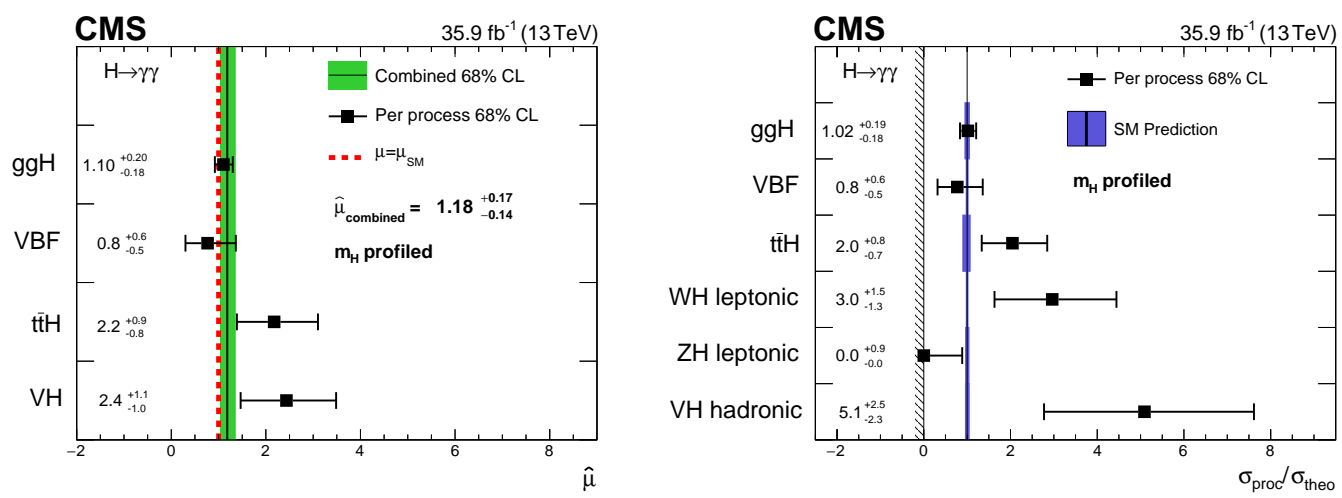

Figure 2: Left: Signal strength modifiers measured for each process (black points), with the SM Higgs boson mass profiled, compared to the overall signal strength modifier (green band) and to the SM expectation (dashed red line). Right: Cross section ratios measured for each process (black points) in the Higgs simplified template cross section framework [32], with the SM Higgs boson mass profiled, compared to the SM expectations and their uncertainties (blue band).

\section{CMS $\mathrm{H} \rightarrow \gamma \gamma$}

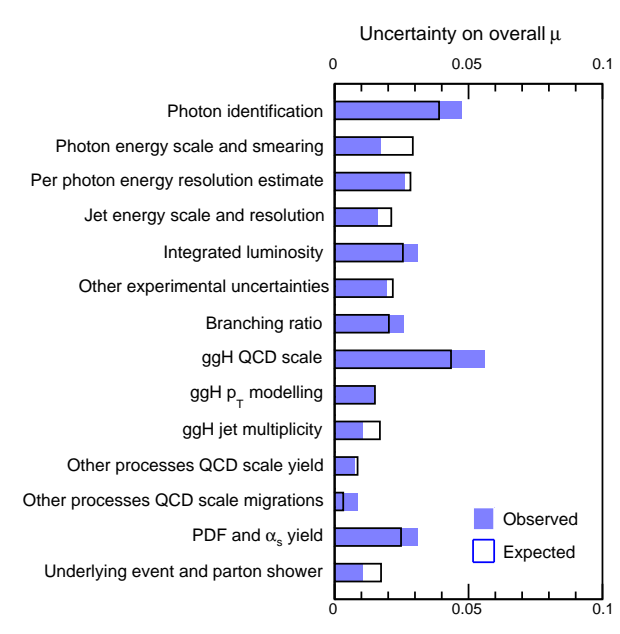

$35.9 \mathrm{fb}^{-1}(13 \mathrm{TeV})$

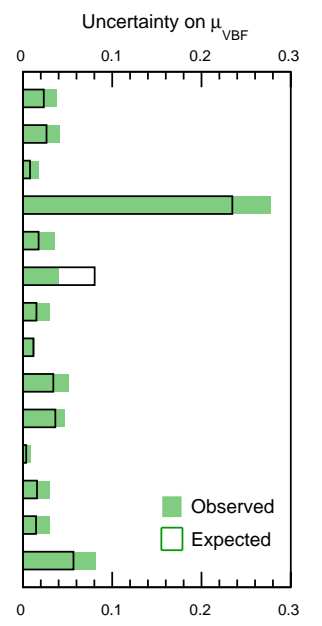

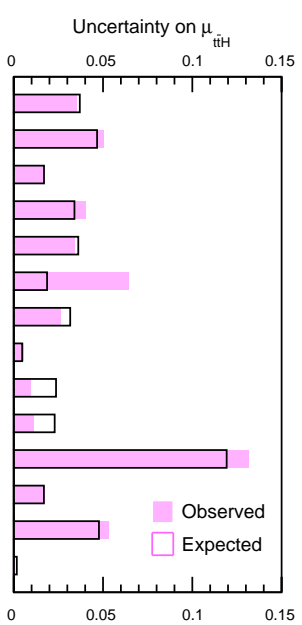

Figure 3: Summary of the impact of the different systematic uncertainties on the overall signal strength modifier and on the signal strength modifiers for the VBF and ttH production processes. The observed (expected) results are shown by the solid (empty) bars.

\section{Results}

A signal model parametric in the Higgs mass $\left(m_{H}\right)$ is constructed from simulated events for each process and event category. Systematic uncertainties of experimental and theoretical origin are included in the signal model. The background model used is the discrete profiling method, where a number of functions are considered as candidates and the choice is treated as a discrete nuisance parameter [3]. To extract results, a maximum likelihood fit to the $m_{\gamma \gamma}$ distribution is performed simultaneously across all categories. The simplest fit performed is for the overall signal strength (mu) relative to the Standard Model (SM) prediction, where all signals are scaled by the same 


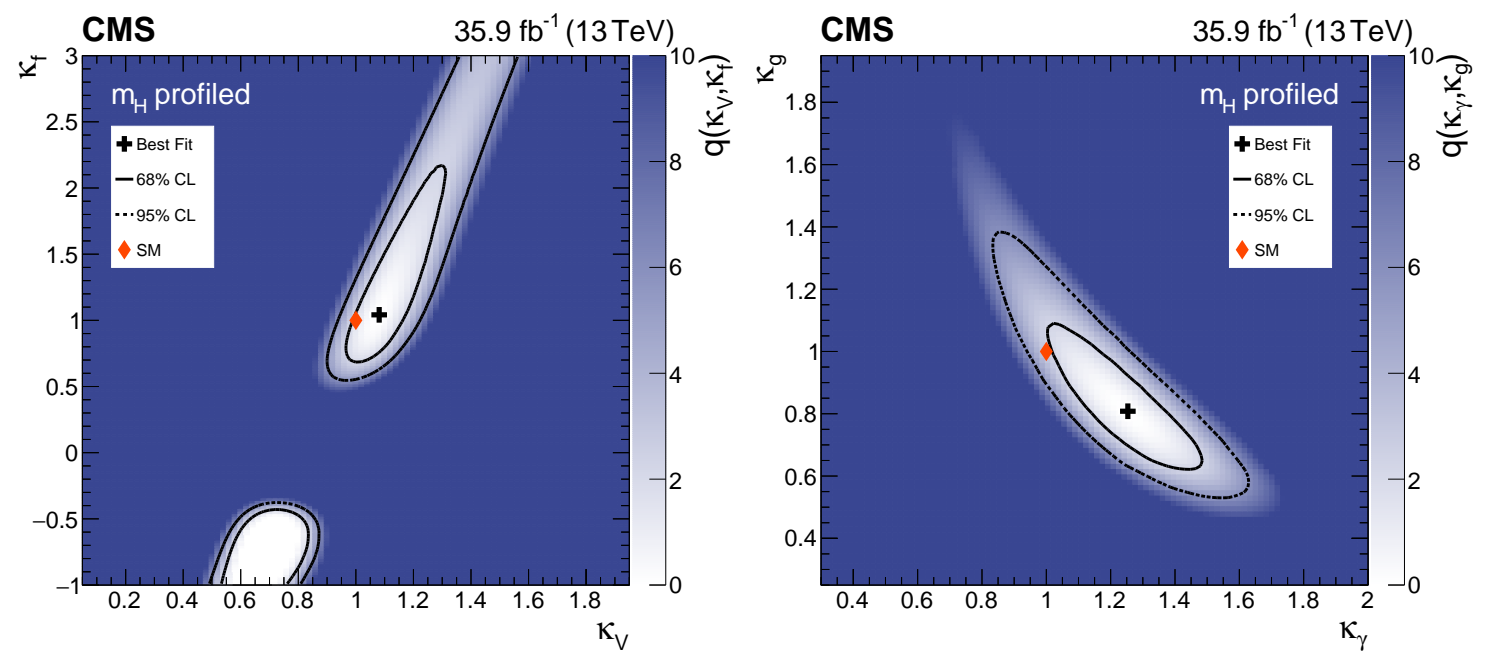

Figure 4: Two-dimensional likelihood scans of $\kappa_{f}$ versus $\kappa_{V}$ on the left, $\kappa_{g}$ versus $\kappa_{\gamma}$ on the right. The cross indicates the best fit value, the diamond indicates the standard model expectation. The colour maps indicate the value of the profile likelihood test statistic, $q$.

factor. The result of the fit is $1.18_{-0.14}^{+0.17}=1.18_{-0.11}^{+0.12}$ (stat $)_{-0.07}^{+0.09}(\mathrm{syst})_{-0.06}^{+0.07}$ (theo), in good agreement with the SM. Additional fits are performed where each production process is scaled independently. The results are shown in Figure 2. A fit at Stage 0 of the Simplified Template Cross-Section framework [4], where the theoretical uncertainties from the overall yield are removed from the fit and included instead on the SM prediction, is also shown in Figure 2.

The impact of systematic uncertainties on the inclusive, VBF and ttH signal strengths are shown in Figure 3. Though the main source of uncertainty is still statistical in origin, the systematic component is of similar size with this dataset. Its importance will increase further with the addition of the 2017 and 2018 datasets.

Finally, results of fits within the so-called $\kappa$-framework are presented. Here the parameters are the coupling strengths of the Higgs boson to other particles normalised to the SM expectation. In Figure 4, two-dimensional scans of both the coupling to fermions and bosons (left) and the effective coupling via loop diagrams to gluons and photons are shown. In each case, good agreement with the SM is observed.

\section{References}

[1] CMS collaboration, S. Chatrchyan et al., The CMS Experiment at the CERN LHC, JINST 3 (2008) S08004.

[2] CMS collaboration, A. M. Sirunyan et al., Measurements of Higgs boson properties in the diphoton decay channel in proton-proton collisions at $\sqrt{s}=13 \mathrm{TeV}, 1804.02716$.

[3] P. D. Dauncey, M. Kenzie, N. Wardle and G. J. Davies, Handling uncertainties in background shapes, JINST 10 (2015) P04015 [1408. 6865].

[4] LHC Higgs Cross Section Working Group collaboration, D. de Florian et al., Handbook of LHC Higgs Cross Sections: 4. Deciphering the Nature of the Higgs Sector, 1610.07922. 